\title{
SUR LES FONCTIONS MÉROMORPHES AUX LIMITES FINES
}

\author{
NOBUSHIGE TODA \\ dédié à Monsieur le Professeur K. Noshiro, à l'occasion \\ de son soixantième anniversaire
}

\section{Introduction}

Dans ce mémoire, on continue d'étudier sur les fonctions à des limites fines que J. L. Doob a trouvées [5]. On a trouvé quelques propriétés des fonctions dans [10]. Dans le paragraphe 2 on donne une condition suffisante afin qu'une fonction admette une limite fine et on étudie sur l'indice harmonique d'un spot asymptotique d'une fonction à une limite fine. Le paragraphe 3 est consacré aux études d'autres propriétés.

\section{Une condition suffisante}

D'abord on donne quelques lemmes.

Lemme 1. Soit $u(z)$ une fonction surharmonique dans un domaine $D$ tel que le point à l'infini $(=\omega)$ est un point-frontière irrégulier de D. Si $u(z) / \log |z|$ est bornée inférieurement dans un voisinage fin de $\omega$, alors $u(z) / \log |z|$ admet une limite fine finie en $\omega$ [2].

Lemme 2. Si un ensemble $E$ quelconque dans le plan est effilé en $\omega$, il existe des circonférences ar̈bitrairement grandes de centre 0 ne rencontrant pas $E$ [4].

Lemme 3. Soit $D$ un domaine comme dans le Lemme 1 et de complémentaire non polaire, $u(z)$ une fonction surharmonique positive dans $D$ et nulle continûment sur les points-frontière réguliers de $D$. Alors il existe une fonction harmonique positive minorante $h(z)$ de $u(z)$ dans $D$ si et seulement si la limite fine de $u(z) / \log |z|$, qui est finie d'après le Lemme 1, est positive.

Démonstration. D'abord on trouve que, s'il existe une fonction $h(z)$ comme dans ce lemme, la limite fine de $h(z) / \log |z|$ (soit $\alpha$ ) est positive. Ce qu'elle

Received March 9, 1966. 
existe est assuré du Lemme 1.

On pose

$$
s(z)= \begin{cases}h(z) & \text { dans } D \\ 0 & \text { ailleurs, }\end{cases}
$$

alors la régularisáe $\dot{s}(z)$ de $s(z)$ est sousharmonique dans le plan fini, de sorte que la moyenne de $\hat{s}(z)$ :

$$
m \hat{s}(r)=\frac{1}{2} \frac{1}{\pi} \int_{0}^{2 \pi} \hat{s}\left(r e^{i \theta}\right) d \theta
$$

est convexe de $\log r$. Donc, la limite

$$
\lim _{r \rightarrow+\infty} \frac{m_{\hat{s}}(r)}{\log r}=\beta
$$

existe. Cette limite est égale à $\alpha$, parce qu'il existe une suite $\left(r_{n}\right)$ telle que $r_{n} \nearrow+\infty,\left(|z|=r_{n}\right) \subset D, h\left(r_{n} e^{i \theta}\right) / \log r_{n}$ tend vers $\alpha$ uniformément par rapport à $\theta$ d'apès le Lemme 2 , et pour cette suite

$$
\beta=\lim _{n \rightarrow \infty} \frac{m_{\hat{s}}\left(r_{n}\right)}{\log r_{n}}=\lim _{n \rightarrow \infty} \frac{h\left(r_{n} e^{i \cap}\right)}{\log r_{n}}=\alpha .
$$

D'après un theorème de $M$. Brelot [3], ce que $\beta$ est finie entraîne que $\hat{s}(z) / \log |z|$ est bornée dans un voisinage de $\omega$. Si $\alpha$ est nulle, grâce à un théorème de $\mathrm{M}$. Brelot [2], $h(z) \equiv 0$. C'est contraire à l'hypothèse, c'est-à-dire, il faut que $\alpha>0$. $u(z) \geq h(z)$, par conséquent, la limite fine de $u(z) / \log |z|$ est positive.

Inversement, si la limite fine de $u(z) / \log |z|$ est positive, $u(z) / G_{w}(z)$ admet une limite fine finie positive en $\omega$, où $G_{w}(z)$ est harmonique positive dans $D$, nulle continûment sur les points-frontière réguliers de $D$ et son flux en $\omega$ est $2 \pi$. En effet, parce que $u(z) / \log |z|$ et $G_{v}(z) / \log |z|$ admettent des limites fines finies positives en $\omega$,

$$
\underset{G_{w}(z)}{u(z)}=\frac{u(z) / \log |z|}{G_{w}(z) / \log |z|}
$$

admet une limite fine finie positive (soit $\delta$ ).

L'ensemble

$$
V=\left(z \in D ; u(z) / G_{w}(z)>(\delta-\varepsilon)\right)
$$

est un voisinage fin de $\omega$, où $\varepsilon$ est un nombre positif plus petit que $\delta$. Par 
conséquent, d'après le Lemme 2, il existe une suite $\left(r_{n}\right)$ telle que $r_{n} \nearrow+\varkappa$, $\left(|z|=r_{n}\right) \subset V: u\left(r_{n} e^{i \theta}\right)>(\grave{o}-\varepsilon) G_{w}\left(r_{n} e^{i \emptyset}\right)$ pour tout $\theta$.

Considérons une fonction surharmonique:

$$
v(z)=u(z)-(\delta-\varepsilon) G_{w}(z)
$$

dans $D \cap\left(|z|<r_{n}\right)=D_{n}, n$ quelconque, alors à un ensemble de capacité zóro sur la frontière de $D_{n}$ près

$$
\lim _{z \rightarrow \partial D_{n}} \inf v(z) \geq 0
$$

Grâce au principe de minimum, $v(z) \geq 0$ dans $D_{n}, n$ quelconque, en conséquence $v(z)$ est non-négative dans $D$. Cela veut dire que $u(z)$ admet une fonction harmonique positive minorante: $(\delta-\varepsilon) G_{w}(z)$.

Soit $f(z)$ une fonction méromorphe dans le plan fini ayant un point singulier essentiel isolé à l'infini, si elle admet une limite fine $\infty$ en $\omega$, il $y$ a un seul spot asymptotique sur $\infty$. On calcule l'indice harmonique de ce spot asymptotique. (Sur la définition d'indice harmonique d'un spot asymptotique, voir [7].)

ThÉoRÈme 1. L'indice harmonique de ce spot asymptotique est 0 ou 1.

Démonstration. Soit $D$ une partie non relativement compacte, conexe de $(z ;|f(z)|>1)$ (il n'exsite qu'un tel domaine $D$ gráce à un théorème de L. Naïm [8]).

On connaît bien l'égalité

$$
\sum_{f\left(z_{0}\right)=\infty} n\left(z_{0}\right) G\left(z, z_{0}\right)+u(z)=\log |f(z)|
$$

dans $D$, où $n\left(z_{0}\right)$ est l'ordre de multiplicité de $f(z)$ en $z_{0}, G\left(z, z_{3}\right)$ est la fonction de Green de $D$ et $u(z)$ est la fonction harmonique minorante de $\log |f(z)|$ la plus grande.

Si $u(z) \neq 0$, on trouve que l'indice harmonique est 1 . D’abord, on démontre que $u(z)=\alpha \cdot G_{w}(z)$ dans $D$ où $\alpha$ est un nombre positif. En effet, comme dans la démonstration du Lemme 3, la limite fine de $u(z) / \log |z|$ est positive finie, par conséquent, $u(z) / G_{w}(z)$ admet une limite fine finie positive (soit $\alpha$ ).

L'ensemble

$$
V=\left(z \in D ;(\alpha+\varepsilon)>u(z) / G_{w}(z)>(\alpha-\varepsilon)\right)
$$

est un voisinage fin de $\omega$, où $\varepsilon$ est un nombre positif quelconque plus petit que $\alpha$, de sorte que, comme dans la démonstration du Lemme 3, on a, dans $D$, 


$$
(\alpha+\varepsilon) G_{w}(z) \geq u(z) \geq(\alpha-\varepsilon) G_{\omega}(z) .
$$

Cela veut dire que

$$
u(z)=\alpha \cdot G_{w}(z)
$$

dans $D$, puisque $\varepsilon$ est positif quelconque. Soit $D_{r}$ une partie non relativement compact connexe de $(z ;|f(z)|>r>1) . \quad D_{r} \subset D$. Alors dans $\mathrm{D}_{r}$ on a une égalité suivante comme dans la première partie de cette démonstration:

$$
\sum_{f\left(z_{0}\right)=\infty} n\left(z_{0}\right) G_{l}\left(z, z_{0}\right)+u_{r}(z)=\log |f(z) / r| .
$$

Puisque le complémentaire de $D_{r}\left(=\mathscr{C} D_{r}\right)$ est effilé en $\omega$ pour tout $r$, d'après le Lemme $3, \log |f(z) / r| / \log |z|$ admet une limite fine finie positive et le Lemme 3 entraîne que $u_{r}(z) \neq 0$. Comme dans la première partie de cette démonstration, $u_{r}(z)=\beta_{r} \cdot G_{w}^{r}(z)$ où $G_{w}^{r}(z)$ est harmonique positive dans $D_{r}$, nulle continûment sur la frontière de $D_{r}$ et son flux en $\omega$ est $2 \pi, \beta_{r}$ un nombre positif. D'après la définition d'indice harmonique, l'indice harmonique de ce spot asymptotique est 1 . Si $u(z) \equiv 0$, l'indice harmonique est évidemment 0 .

N. B. Cette démonstration indique que l'indice harmonique est 0 ou 1 si pour un $r, u_{r}(z) \equiv 0$ ou non réspectivement.

Maintenant, on donne une condition suffisante pour qu'une fonction admette une limite fine en $\omega$.

Soit $f(z)$ une fonction méromorphe dans le plan fini ayant un point singulier essentiel isolé à l'infini, $D$ une partie non relativement compacte, connexe de $(z ;|f(z)|>1), G\left(z, z_{0}\right)$ la fonction de Green de $D$. On connaît bien l'égalité suivante

$$
\sum_{f\left(z_{0}\right)=\infty} n\left(z_{0}\right) G\left(z, z_{0}\right)+u_{w}(z)=\log |f(z)|
$$

dans $D$, où $n(z)$ est l'ordre de multiplicité de $f(z)$ en $z, u_{w}(z)$ la fonction harmonique minorante de $\log |f(z)|$ la plus grande. Dans cette situation, on a

THÉORÈME 2. Si $u_{w}(z) \neq 0$ et $s i$

$$
\lim _{r \rightarrow+\infty} \frac{m(r, f)}{\log r}<+\infty,
$$

alors $f(z)$ admet une limite fine $\infty$ en $\omega$.

Démonstration, On pose 


$$
u(z)= \begin{cases}u_{\omega}(z) & \text { dans } D \\ 0 & \text { ailleurs }\end{cases}
$$

alors $u(z)$ est sousharmonique non-négative dans le plan fini.

Soit

$$
m_{u}(r)=\frac{1}{2 \pi} \int_{0}^{2 \pi} u\left(r e^{i \theta}\right) d \theta
$$

la moyenne de $u(z) . \quad m_{u}(r)$ est convexe de $\log r$, par conséquent, $m_{u}(r) / \log r$ admet une limite pour $r \nearrow+\infty$ qui est finie dans ce cas-1̀, parce que

$$
m_{u}(r) \leq m(r, f)
$$

pour tout $r$. Cela veut dire qu'en vertu d'un théorème de M. Brelot [3], $u(z) / \log |z|$ est bornée dans un voisinage de $\omega$. En considérant que $u_{w}(z) \neq 0$, le point à l'infini est un point irrégulier de $D$ gráce à un théorème de $M$. Brelot [2]. Cela veut dire que $\mathscr{C} D$ est effilé en $\omega$, par conséquent, $f(z)$ admet une limite fine en $\omega$, qui est $\propto$ en vertu d'un théorème de M. Heins [6].

UN EXEMPLE. Soit $f(z)$ une fonction méromorphe dans le plan ayant un point singulier essentiel isolé à l'infini et admettant une limite fine 0 en ce point. Alors $g(z)=f(z)+z$ admet une limite fine $\infty$ en $\omega$, parce que $|z|-|f(z)| \leq|g(z)| . \log |g(z)| / \log |z|$ a une limite fine 1 en $\omega$, par conséquent, d'après le Lemme 3, l'indice harmonique du spot asymptotique sur $\infty$ est 1. Cette fonction est aussi un exemple qui satisfait les conditions du Théorème 2.

N. B. On ne peut pas donner un exemple dont l'indice harmonique est 0 .

\section{Direction de Julia}

Une fonction méromorphe dans le plan ayant un point singulier essentiel isolé en $\omega$ et admettant une limite fine en $\omega$ a une propriété intéressante pour la direction de Julia. On le voit suivant.

Lemme 4. Soit $E$ un ensemble compact dont le complémentaire $\mathscr{C} E$ est un domaine, $0<\lambda<1, E_{n}=E \cap\left(\lambda^{n+1} \leq|z| \leq \lambda^{n}\right), C_{n}$ capacité de $E_{n} . \quad$ Si

$$
\sum_{n=1}^{\infty} \frac{C_{n}}{\lambda^{n}}=+\infty
$$

l'origine 0 est un point régulier du domaine $\mathscr{C} E$ (voir [11]). 
En utilisant ce Lemme, on démontre un autre lemme. Soit $D$ un domaine tel que l'origine est un point-frontiére de $D, E=\mathscr{C} D$,

$$
K_{r}=(\theta ;(\arg z=\theta) \cap(E-\{0\}) \cap(|z| \leq r) \neq \phi) \text { et } \theta_{r}=\int_{K_{r}} d \theta .
$$

Lemme 5. Si l'origine 0 est un point irrégulier du domaine $D, \lim _{r \rightarrow 0} \theta_{r}=0$.

Démonstration. On peut supposer que $E$ soit compact. Soit $K_{n}=(\theta$; $\left.(\arg z=\theta) \cap E_{n} \neq \phi\right), \theta_{n}=\int_{K_{n}^{\prime}} d \theta$.

1) le cas où $(E-\{0\}) \subset(0 \leq \arg z \leq \pi / 2)$. On sait que si $\Gamma$ est un arc sur $|z|=R$ dont l'angle vu de l'origine est $\theta$, la capacité de $\Gamma$ est $R \cdot \sin \theta / 4$. Alors en considérant la définition de capacité par le diamètre transfini, on obtient l'inégalité

$$
\lambda^{n+1} \sin \theta_{n} / 4 \leq C_{n} .
$$

Grâce au Lemme 4 , en utilisant $2 \theta / \pi \leq \sin \theta$ pour $\theta$ entre 0 et $2^{-1} \pi$, pour $\varepsilon$ positif quelconque, il existe un $n_{0}$ tel que

$$
\frac{\lambda}{2 \pi} \theta_{\lambda} n_{0} \leq \frac{\lambda}{2 \pi} \sum_{n=n_{0}}^{\infty} \theta_{n} \leq \sum_{n=n_{0}}^{\infty} \frac{C_{n}}{\lambda} \leq \varepsilon .
$$

Cela veut dire que

$$
\lim _{r \rightarrow 0} \theta_{r}=0
$$

2) les autres. Soit

$$
E_{i}=(E-\{0\}) \cap\left(\frac{\pi}{2}(i-1) \leq \arg z \leq \frac{\pi}{2} \cdot i\right) \cup\{0\} \quad i=1,2,3,4,
$$

alors $E=\bigcup_{i=1}^{4} E_{i}$. Pour chaque $E_{i}$, on applique le cas (1) et on obtient le résultat.

THÉoR̀̀me 3. Soit $f(z)$ une fonction méromorphe dans le plan fini ayant un point singulier essentiel isolé à l'infini et admettant une limite fine en ce point. Alors $f(z)$ a une direction de Julia telle que dans n'importe quel domaine angulaire qui est partagé en deux domaines angulaires égales par cela, $f(z) n^{\prime} a$ pas de valeurs exceptionnelles au sens de Picard.

Démonstration. On peut supposer que la limite fine soit 0 . Soit $D$ une partie non relativement compacte, connexe de $(z ;|f(z)|<1), D_{\varepsilon}=\left(z \in D ; G\left(z, z_{0}\right)>\frac{\varepsilon}{2}\right)$ 
où $G\left(z, z_{0}\right)$ est la fonction de Green de $D$ et $\lim _{z \rightarrow \infty} \sup _{z \rightarrow} G\left(z, z_{0}\right)=\varepsilon$ est positif parce que $\mathscr{C} D$ est effilié en $\omega$. $\mathscr{C} D_{\varepsilon}$ est aussi effilé en $\omega$. Soit $\nu_{f}^{\circ}(w)$ le nombre de points de $D_{\varepsilon}$ où $f(z)$ est égale à $w, w$ quelconque de la sphère de Riemann. Alors comme dans la démonstration du théorème $3[9], \nu f(w)$ est bornée et $f(z)$ tend vers 0 sur $D_{\varepsilon}$ quand $z$ tend vers $\omega$. $\mathscr{C} D_{s}$ se compose de domaines de nombre infini dont les frontières sont analytiques. Soit $A_{n}$ un domaine com. posant connexe de $\mathscr{C} D_{\varepsilon}$ qui contient au moins un zéro de $f(z)$. Il existe un nombre infini de tels domaines parce que $f(z)$ n'a pas de valeurs exceptionnelles au sens de Picard d'après un théorème de J. L. Doob [5] et que $\nu_{f}^{\varepsilon}(0)$ est fini. Soit $a_{n}=r_{n} e^{i \theta_{n}}$ un des zéro de $f(z)$ dans $A_{n}$. On peut supposer que $r_{1} \leq r_{2} \leq r_{3} \leq \cdots \leq r_{n}, \lambda+\infty$, Soit $\theta_{0}$ un des points d'accumulation de $\left(\theta_{n}\right)$, $\beta_{n}$ l'angle de $A_{n}$ vu de l'origine 0 . Alors en considérant l'inverse de $A_{n}$ par rapport ̀̀ $|z|=1[1]$, grace au Lemme $5, \beta_{n}$ tend vers $0 . \operatorname{Arg} z=\theta_{0}$ est une direction que l'on cherche. En effet, soit $\Delta$ un domaine angulaire arbitraire qui contient arg $z=\theta_{0}$ comme ligne droite qui le partage en deux parties égales:

$$
\Delta=\left(z ; \theta_{0}-\varepsilon<\arg z<\theta_{0}+\varepsilon\right)
$$

où $\varepsilon$ est un nombre positif quelconque. Puisque $\beta_{n}$ tend vers 0 , il existe un $n_{0}$ tel que pour tout $n$ plus grand que $n_{0}, A_{n} \subset \Delta$. (On peut supposer que $\theta_{n}$ tend vers $\theta_{0 .}$ )

On trouve maintenant que sur $A=\bigcup_{n=1}^{\infty} A_{n}$, il n'existe pas de valeurs exceptionnelles au sens de Picard. Pour $a \neq 0, \infty$, en considérant que sur $D_{\varepsilon} f(z)$ tend vers 0 , il existe un $n_{a}$ tel que pour tout $n \geq n_{a}$,

$$
f^{-1}\left(|w|>|a|-\varepsilon_{1}\right) \subset A_{n}
$$

où $\varepsilon_{1}$ est un nombre positif plus petit que $|a|$. C'est-ł̇-dire que $f(z)$ admet a dans $A_{n}\left(n \geq n_{a}\right)$ au moins une fois. On a pris $A_{n}$ de la manière à contenire au moins un zéro et un pôle de $f(z)$ dans $A_{n}$. On a le résultat.

\section{Bibliographie}

[1] M. Brelot, Sur le rôle du point à l'infini dans la thóorie des fonctions harmoniques. Ann. E. N. S. 61 (1944), 301-332.

[2] - Étude générale des fonctions harmoniques ou surharmoniques positives au voisinage d'un point-frontière irrégulier. Ann. Univ. Grenoble, 22 (1946), 201-219.

[3] - Étude des fonctions sousharmoniques au voisinage d'un point singulier. Ann. 
Inst. Fourier, 1 (1949), 121-156.

[ 4 ] - Éléments de la théorie classique du potentiel. Paris, C. D. U. $3^{e}$ édition 1965.

[5] J. L. Doob, Some classical function theory theorems and their modern versions. Ann. Inst. Fourier, 15 (1965), 113-135.

[6] M. Heins, On the Lindelöf principle. Ann. Math. 61 (1955), 440-473.

[ 7 ] - Asymptotic spots of entire and meromorphic functions. Ann. Math. 66 (1957), 430-439.

[8] L. Naïm, Sur le ròle de la frontière de R. S. Martin dans la théorie du potentiel. Ann. Inst. Fourier, 7 (1957), 183-281.

[9] N. Toda, Étude des fonctions méromorphes au voisinage d'un point-frontière irrégulier. Bull. Sciences Math. $2^{\ominus}$ série, 89 (1965), 93-102.

[10] --, Sur l'allure des fonctions méromorphes. Nagoya Math. J. 26 (1966), 173-181.

[11] M. Tsuji, Potential theory in modern function theory. Maruzen, Tokyo 1959.

Institut de mathématiqus

Université de Nagoya 